\title{
BMJ Open Prognostic role of microRNA-205 in multiple human malignant neoplasms: a meta-analysis of 17 studies
}

\author{
Jia-yi Zhang, ${ }^{1}$ Meng-yan Sun, ${ }^{2}$ Ning-hong Song, ${ }^{1}$ Zhong-lei Deng, ${ }^{3}$ Chun-yu Xue,${ }^{2}$ \\ Jie Yang ${ }^{1}$
}

To cite: Zhang J-yi, Sun Myan, Song N-hong, et al. Prognostic role of microRNA205 in multiple human malignant neoplasms: a meta-analysis of 17 studies. BMJ Open 2015;5:e006244. doi:10.1136/bmjopen-2014006244

- Prepublication history for this paper is available online. To view these files please visit the journal online (http://dx.doi.org/10.1136/ bmjopen-2014-006244)

$\mathrm{J}-\mathrm{yZ}$ and M-yS are joint first authors and contributed equally.

Received 29 July 2014 Revised 2 December 2014 Accepted 15 December 2014

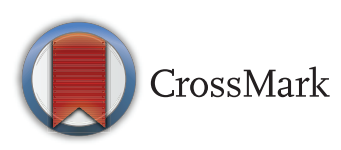

${ }^{1}$ From Department of Urology, The First Affiliated Hospital Of Nanjing Medical University, Nanjing, Jiangsu, China

${ }^{2}$ From Department of Plastic Surgery, Changhai Hospital Affiliated To Second Military Medical University, Shanghai, China

${ }^{3}$ From Department of

Urology, Affiliated Hospital of Nanjing Medical University of TCM, Nanjing, Jiangsu, China

Correspondence to

Dr Jie Yang;

yj197912@163.com

\section{ABSTRACT}

Objective: MicroRNA-205 (miRNA-205) was revealed as an attractive prognostic tumour biomarker in recent studies. However, the results of different studies have been inconsistent. We conducted a meta-analysis to elucidate the precise predictive value of miRNA-205 in various human malignant neoplasms.

Design: Meta-analysis.

Data sources: Qualified studies were identified up to 5 June 2014 by performing online searches in PubMed, EMBASE and Web of Science, and additional quality evaluations.

Participants: Seventeen eligible studies with 4827 patients were ultimately enrolled in this meta-analysis. Outcome measures: The heterogeneity between studies was assessed using I ${ }^{2}$ statistics. Pooled HRs with $95 \%$ Cls for patient survival and disease recurrence were calculated to investigate the correlation between miRNA-205 expression and cancer prognosis.

Results: Our results indicate that elevated miRNA-205 was significantly associated with enhanced overall survival in the breast cancer subgroup ( $\mathrm{HR}=0.78,95 \%$ $\mathrm{Cl} 0.67$ to 0.91 ) and superior disease-free survival/ recurrence-free survival in the adenocarcinoma subgroup (HR=0.68, 95\% $\mathrm{Cl} 0.49$ to 0.94 ).

Conclusions: miRNA-205 is a promising biomarker for predicting the recurrence and progression of patients with adenocarcinomas or breast cancer. Owing to its complex roles, further relevant studies are warranted.

\section{INTRODUCTION}

MicroRNAs (miRNAs), which belong to a class of highly conserved and small noncoding RNAs, are approximately 22-25 nucleotides in length and abundant in animals, plants and even in viruses. ${ }^{12}$ Mature miRNAs negatively regulate target gene expression at the post-transcriptional level by binding to the 3 '-untranslated complementary sequence of target mRNA. Thus, miRNAs play vital roles in various biological processes, such as the cell cycle, apoptosis, proliferation and differentiation. ${ }^{3-6}$ A newly identified
Strengths and limitations of this study

- This is the first meta-analysis reporting the prognostic value of microRNA-205 (miRNA-205) for cancers in the medical literature.

- A broad search strategy was used with few restrictions to minimise any potential publication bias.

- The retrospective nature of the data collection process increased the risk of selection bias in the studies.

- For subgroup analysis, elevated miRNA-205 was significantly associated with enhanced overall survival in the breast cancer subgroup and superior disease-free survival/recurrence-free survival in the adenocarcinoma subgroup.

- The major limitation of our analysis involves the inclusion of a relatively small number of studies on Asians, which might have decreased the applicability of our results across different ethnicities.

function for miRNAs has also been reported, namely, the regulation of gene expression at the transcriptional level. ${ }^{7}$ Therefore, miRNAs are considered to be diagnostic or prognostic biomarkers of carcinomas. ${ }^{8-10}$

miRNA-205 is one of the most frequently studied miRNAs, exhibiting aberrant expression in multiple human malignancies compared with their normal counterparts. The majority of studies have demonstrated that miRNA-205 is a tumour suppressor. ${ }^{5} 68$ 11-17 For instance, Markou et al reported that breast cancer cell lines express reduced miRNA-205 levels compared with nonmalignant cells. The ectopic expression of miRNA-205 significantly inhibits cell proliferation, growth and invasion, whereas downregulated miRNA-205 expression is associated with reductions in disease-free survival (DFS) and overall survival (OS) during the early stages of breast cancer. ${ }^{6}$ miRNA-205 expression is also significantly reduced in glioma tissues compared with non-neoplastic brain tissues. In addition, multivariate analyses have demonstrated that miRNA-205 expression is 
an independent prognostic indicator of OS in patients with glioma. ${ }^{8}$ However, certain studies have presented contradictory results, suggesting that miRNA-205 may function as an oncogene. ${ }^{18-23}$ Karaayvaz et al have confirmed that the expression of miRNA-205 is significantly increased in endometrial tumours compared with normal tissues. Kaplan-Meier survival curve analysis revealed the association of increased miRNA-205 expression with poor OS. ${ }^{21}$ Moreover, a dynamic change in the prognostic value of miRNA-205 expression was observed between preoperative and postoperative patients with lung carcinoma. ${ }^{24}$

Although various studies have reported contradictory results, miRNA-205 is undeniably an attractive mediator of specific target genes that play important roles in tumour initiation and progression. However, given its complex roles, it remains unclear whether miRNA-205 acts as a tumour suppressor or an oncogene. Therefore, we conducted this systematic review using meta-analysis to shed light on the relationship between miRNA-205 expression and the prognosis of patients with carcinoma. We also discussed the possibility of utilising miRNA-205 as a prognostic marker in clinical applications.

\section{METHODS}

\section{Search strategy}

We searched online databases, including PubMed, EMBASE and Web of Science, to identify relevant literature published until 5 June 2014. For the literature retrieval, combinations of the keywords 'microRNA-205', 'microrna-205', 'miRNA-205', 'miRNA-205', 'cancer', 'carcinoma', 'neoplasm', 'tumour', 'tumor', 'overall survival', 'recurrence' and 'prognosis' were utilised. The following criteria were used to select the published studies: (1) English publications; (2) a focus on patients with malignancies and (3) the association of miRNA-205 expression with cancer prognosis. In addition, we searched for studies published in Chinese to comprehensively understand the role of miRNA-205 in cancer.
However, no Chinese studies were available regarding the association of miRNA-205 expression with cancer prognosis.

\section{Quality assessment}

This meta-analysis was strictly performed according to the preferred reporting items of the systematic reviews and meta-analysis (PRISMA) statement. ${ }^{25}$ The key points of the quality assessment included clear definitions of the following: (1) the study population and country of origin, (2) the type of carcinoma, (3) the study design, (4) the outcome assessment, (5) the detection of miRNA-205, (5) the miRNA-205 cut-off value and (6) a sufficient follow-up period. Studies were excluded when they did not meet all of the above criteria, to maintain the quality of the meta-analysis. Sensitivity analyses were also performed to avoid biases in the results due to certain low-quality studies (figures 1A, B). Moreover, we used the Cochrane Risk of Bias tool and graded each potential source of bias as a low, high or unclear risk (http://www.cochrane-handbook.org; figures 2 and 3). A flow diagram with details of the study selection process is presented in figure 4 .

\section{Data extraction}

All eligible studies were identified by J-yZ and M-yS, and uncertain data were reassessed by JY. The extracted data elements included the following: (1) the first author's name and year of publication; (2) the study population, nationality, ethnicity, type of disease, pathology and detected sample; (3) the detection method, cut-off definition and follow-up time; (4) HR associated with downregulated miRNA-205 expression for OS, recurrence-free survival (RFS), and DFS along with their 95\% CI and $\mathrm{p}$ values and (5) the median or mean age of the patients. If only Kaplan-Meier curves were available, data were extracted from graphical survival plots to extrapolate HRs with 95\% CIs using previously described methods. ${ }^{26} 27$ All of the aforementioned data are comprehensively detailed in tables 1 and 2.
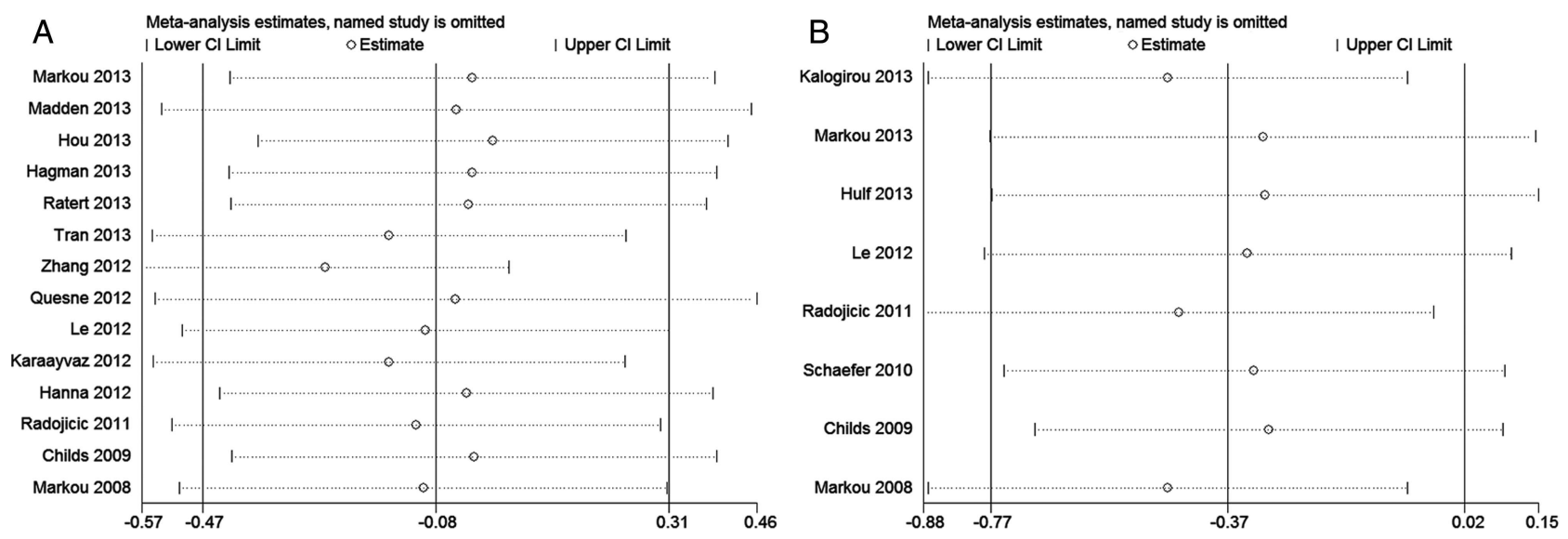

Figure 1 Sensitivity analysis of each included study. (A) Sensitivity analysis of overall survival for individual studies.

(B) Sensitivity analysis of disease-free survival/relapse-free survival for individual studies. 


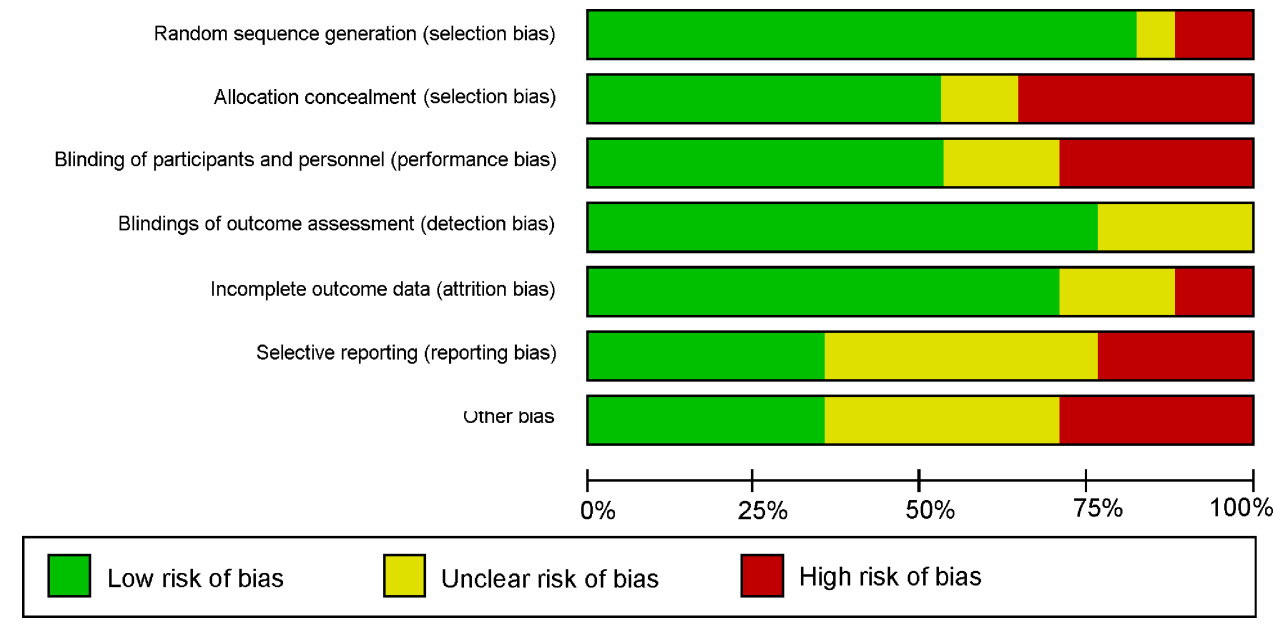

Figure 2 Risk of bias graph. Review authors' judgements for each risk of bias item are presented as percentages across all included studies.

\section{Statistical analysis}

The fixed-effects model (Mantel-Haenszel method) or the random-effects model (DerSimonian-Laird method) was

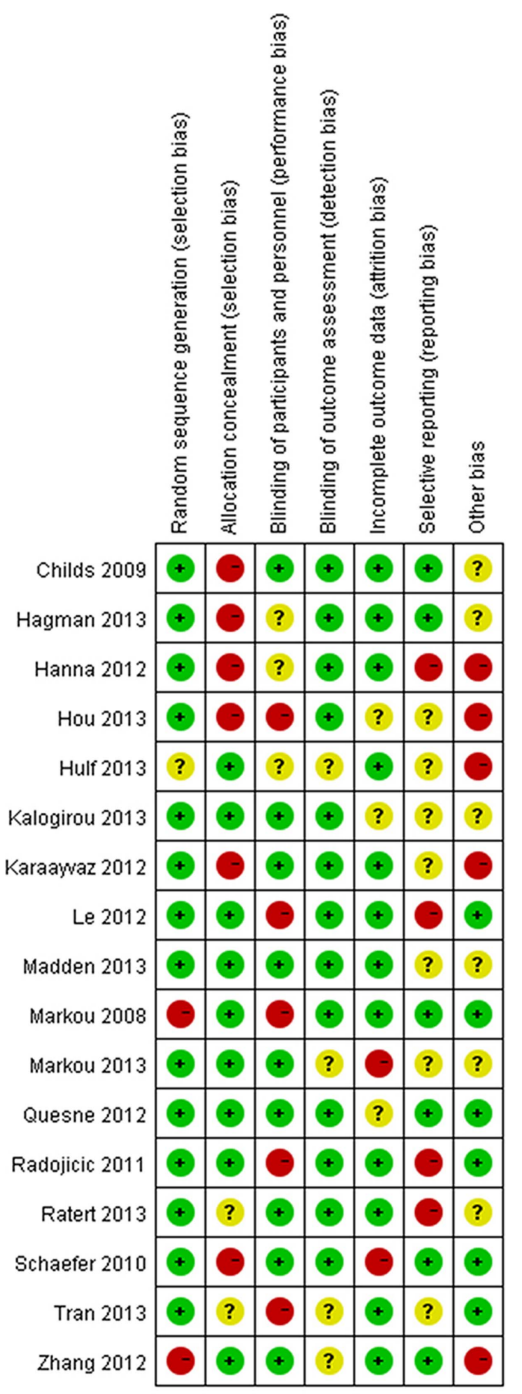

Figure 3 Risk of bias summary. Review authors' judgements for each risk of bias item for each included study. used for meta-analysis according to the heterogeneity among the pooled studies. ${ }^{28}$ The heterogeneity test for pooled HRs was verified using the Cochran $Q$ test and quantified using the Higgins $\mathrm{I}^{2}$ statistic. If significant heterogeneity was observed ( $p<0.10$ or $\mathrm{I}^{2}>50 \%$ ), a random-effects model was applied; otherwise, the fixed-effects model was utilised. Moreover, we minimised the influence of heterogeneity by classifying the enrolled studies into subgroups based on similar characteristics. The publication bias was estimated using Egger's linear regression test with a funnel plot. ${ }^{29}$ All $\mathrm{p}$ values were calculated using a two-sided test, and a $p$ value of less than 0.05 was considered statistically significant. All statistical analyses were conducted with Stata12 (StataCorp LP, College Station, Texas, USA), Review Manager (RevMan) V.5.3 (Copenhagen: the Nordic Cochrane Centre, the Cochrane Collaboration, 2014) and Microsoft Excel (V.2007, Microsoft Corporation, Redmond, Washington, USA).

\section{RESULTS}

\section{Summary of enrolled studies}

A total of 415 studies were collected from a primary literature survey using PubMed, EMBASE and Web of Science, and 346 records were excluded because they were review articles, letters, non-English articles, did not use human subjects, or were not relevant to the current analysis. Of the remaining studies under evaluation, 27 did not contain sufficient survival data (HRs and survival curves), 13 were not directly related to the specific outcomes, 7 did not use tumour tissues or blood samples, 4 did not report dichotomous outcomes for miRNA-205 expression and 1 had overlapping data sets. Finally, 17 studies were considered eligible for this meta-analysis ${ }^{58} 8^{11-24}$ (figure 4).

The main features of the 17 enrolled studies are systematically summarised in tables 1 and 2. Nine studies reported patient OS, three focused on RFS and five investigated OS as well as DFS. We collected data from these 17 studies, which included 4892 participants. Of these studies, 14 focused on Caucasians and 3 evaluated 


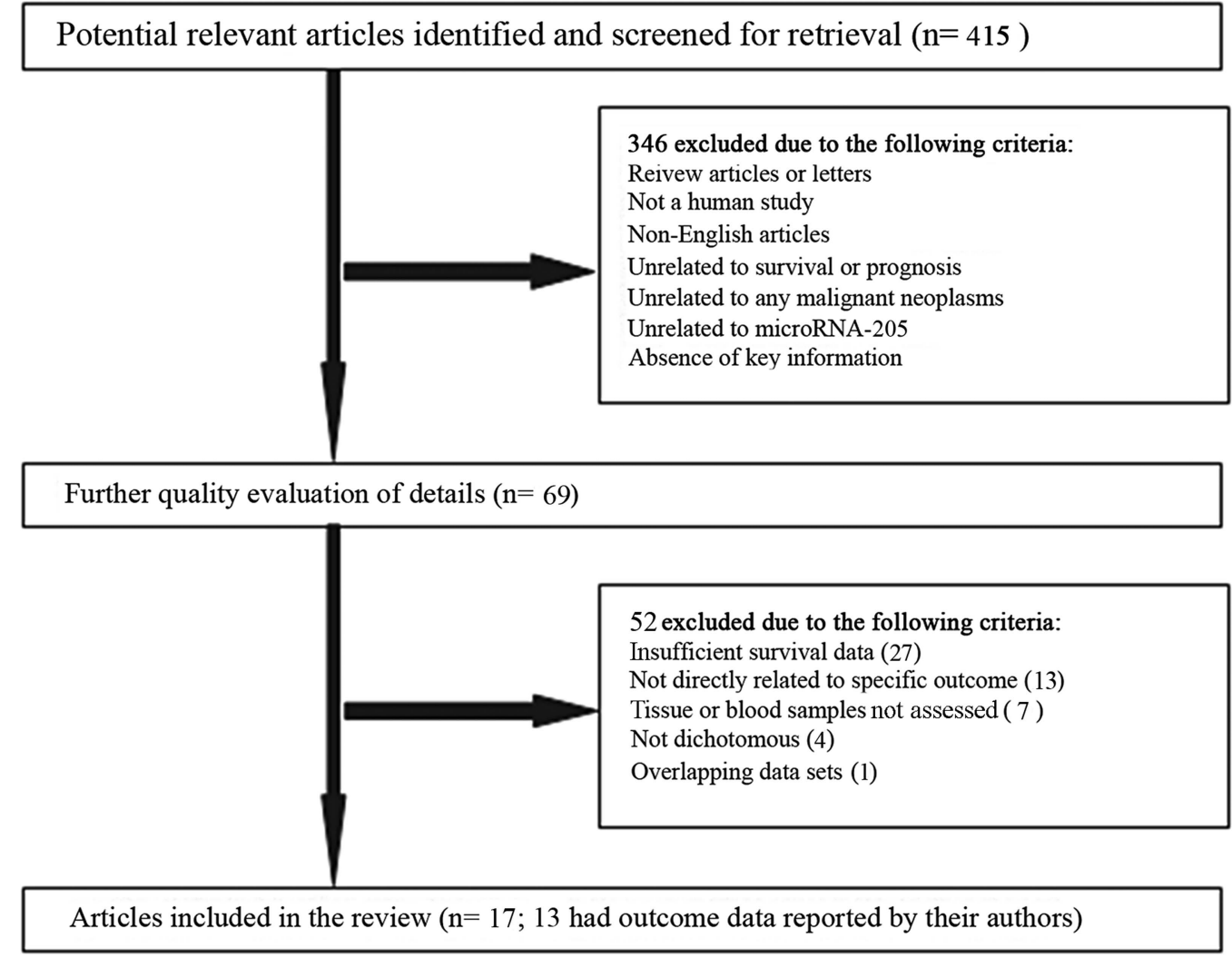

Figure 4 Flow diagram of the study selection process.

Asians. All of these studies were retrospective in design except for one, which was prospective. The malignant neoplasms assessed in these studies included breast cancer, bladder cancer, endometrial cancer, head and neck cancer, glioma, melanoma, non-small cell lung cancer and prostate cancer. Twelve studies focused on the pathological type of adenocarcinoma (adenoCA), two assessed squamous carcinoma $(\mathrm{SqCa})$, two evaluated combined carcinomas (adenoCA and SqCa) and one focused on glioblastomas. Tissue samples were used to determine miRNA-205 expression in all studies except one, which detected expression in serum samples. Quantitative real-time PCR (qRT-PCR) was widely used in 14 studies to assess miRNA-205 expression, in situ hybridisation was performed in 2 studies and microarray analysis was conducted in 1 study.

\section{OS associated with miRNA-205 expression}

A total of 14 studies were used for OS analysis (table 3 and figure 5) with a random-effects model due to significant heterogeneity $\left(\mathrm{p}<0.001, \mathrm{I}^{2}=76.8 \%\right)$. Our results failed to demonstrate any significant association between miRNA-205 expression and OS. Moreover, stratified analyses were performed using studies with the same characteristics (table 3, figures 5 and 6). However, only a pooled analysis of four studies in the breast cancer subgroup indicated that increased miRNA-205 expression was significantly correlated with enhanced OS (pooled $\mathrm{HR}=0.78,95 \%$ CI 0.67 to 0.91 ), which was determined using a fixed-effects model given the low heterogeneity among the studies $\left(p=0.199, I^{2}=35.5 \%\right)$. No other subgroups exhibited any significant results as shown by stratified analyses.

\section{Recurrence associated with miRNA-205 expression}

A total of eight studies included in the DFS/RFS analysis revealed a protective role of increased miRNA-205 expression (pooled $\mathrm{HR}=0.69$, 95\% CI 0.46 to 1.03 ), as determined by a random-effects model $\left(\mathrm{p}=0.079, \mathrm{I}^{2}=45.1 \%\right.$; table 3 and figure 5). In the stratified analyses, increased miRNA-205 expression was significantly associated with enhanced DFS/RFS in the adenoCA subgroup (pooled $\mathrm{HR}=0.68,95 \%$ CI 0.49 to 0.94 ), as determined by a fixed-effects model $\left(\mathrm{p}=0.116, \mathrm{I}^{2}=45.9 \%\right)$. However, no significant association was observed for any other subgroup (table 3 , figures 5 and 6 ).

\section{Sensitivity analyses}

In the OS and DFS/RFS studies, our sensitivity analyses did not indicate alterations in the results due to the inclusion of any individual study (figure 1A,B), suggesting that no single study significantly influenced the pooled HR or the $95 \%$ CI.

\section{Publication bias}

The funnel plots of the publication bias are presented in figure $5 \mathrm{C}, \mathrm{D}$. In the pooled analyses of OS and DFS/ RFS, the Egger's test $p$ values were 0.720 and 0.581, 
Table 1 Main characteristics of studies included in the meta-analysis

\begin{tabular}{|c|c|c|c|c|c|c|c|c|c|c|}
\hline $\begin{array}{l}\text { First author, } \\
\text { publication year }\end{array}$ & $\begin{array}{l}\text { Case } \\
\text { nationality }\end{array}$ & $\begin{array}{l}\text { Median or } \\
\text { mean age }\end{array}$ & $\begin{array}{l}\text { Dominant } \\
\text { ethnicity }\end{array}$ & $\begin{array}{l}\text { Study } \\
\text { design }\end{array}$ & $\begin{array}{l}\text { Malignant } \\
\text { disease }\end{array}$ & $\begin{array}{l}\text { Main type } \\
\text { of pathology }\end{array}$ & $\begin{array}{l}\text { Detected } \\
\text { sample }\end{array}$ & $\begin{array}{l}\text { Survival } \\
\text { analysis }\end{array}$ & $\begin{array}{l}\text { Source } \\
\text { of HR }\end{array}$ & $\begin{array}{l}\text { Maximum } \\
\text { months of } \\
\text { follow-up }\end{array}$ \\
\hline Markou, 2013 & Greece & $60^{\text {Median }}$ & Caucasian & $\mathrm{R}$ & Breast cancer & AdenoCA & Tissue & OS/ DFS & Reported & 140 \\
\hline Madden, 2013 & Ireland & NM & Caucasian & $\mathrm{R}$ & Breast cancer & AdenoCA & Tissue & OS & Reported & 175 \\
\hline Kalogirou, 2013 & Germany & $63^{\text {Median }}$ & Caucasian & $\mathrm{R}$ & Prostate cancer & AdenoCA & Tissue & bRFS & Reported & 154 \\
\hline Hulf, 2013 & Australia & $61^{\text {Median }}$ & Caucasian & $\mathrm{R}$ & Prostate cancer & AdenoCA & Tissue & RFS & Reported & 150 \\
\hline Hou, 2013 & China & $45^{\text {Mean }}$ & Asian & $\mathrm{R}$ & Human glioma & Glioblastomas & Tissue & OS & Reported & 60 \\
\hline Hagman, 2013 & Sweden & NM & Caucasian & $\mathrm{R}$ & Prostate cancer & AdenoCA & Tissue & OS & Reported & 204 \\
\hline Ratert, 2013 & Germany & NM & Caucasian & $\mathrm{R}$ & Bladder cancer & AdenoCA & Tissue & Os & SC & 161 \\
\hline Tran, 2013 & USA & NM & Caucasian & $\mathrm{R}$ & Bladder cancer & AdenoCA & Tissue & OS & SC & 150 \\
\hline Zhang, 2012 & China & $60^{\text {Mean }}$ & Asian & $\mathrm{R}$ & NSCLC & $\mathrm{SqCa}$ & Tissue & OS & Reported & 16.25 \\
\hline Quesne, 2012 & UK & NM & Caucasian & $\mathrm{R}$ & Breast cancer & AdenoCA & Tissue & OS & Reported & 120 \\
\hline Le, 2012 & China & $60^{\text {Mean }}$ & Asian & $\mathrm{R}$ & NSCLC & AdenoCA/SqCa & Serum & OS/DFS & Reported & 30 \\
\hline Karaayvaz, 2012 & USA & NM & Caucasian & $\mathrm{R}$ & Endometrial cancer & AdenoCA & Tissue & OS & Reported & 150 \\
\hline Hanna, 2012 & USA & $63.43^{\text {Mean }}$ & Caucasian & $\mathrm{R}$ & Melanoma & AdenoCA & Tissue & OS & Reported & 120 \\
\hline Radojicic, 2011 & Greece & NM & Caucasian & $\mathrm{R}$ & Breast cancer & AdenoCA & Tissue & OS/DFS & SC & 120 \\
\hline Schaefer, 2010 & Germany & $63^{\text {Median }}$ & Caucasian & $\mathrm{R}$ & Prostate cancer & AdenoCA & Tissue & RFS & Reported & 93 \\
\hline Childs, 2009 & USA & $60^{\text {Mean }}$ & Caucasian & $P$ & HNC & $\mathrm{SqCa}$ & Tissue & OS/DFS & Reported & 60 \\
\hline Markou, 2008 & Greece & $60^{\text {Median }}$ & Caucasian & $\mathrm{R}$ & NSCLC & AdenoCA/SqCa & Tissue & OS/DFS & SC & 50 \\
\hline
\end{tabular}

Study design is described as prospective $(P)$ or retrospective $(R)$.

AdenoCA, adenocarcinoma; bRFS, biochemical recurrence-free survival; DFS, disease-free survival; HNC, head and neck cancer; NM, not mentioned; NSCLC, non-small cell lung cancer;

OS, overall survival; RFS, relapse-free survival; SC, survival curve; SqCa, squamous carcinoma. 
Table 2 HRs and 95\% Cls for patient survival or disease progression in association with miRNA-205 expression in enrolled studies

\begin{tabular}{|c|c|c|c|c|c|c|c|c|}
\hline \multirow[b]{2}{*}{ First author, publication year } & \multirow[b]{2}{*}{$\begin{array}{l}\text { Main assay } \\
\text { method }\end{array}$} & \multirow[b]{2}{*}{ Cut-off value } & \multicolumn{2}{|c|}{ Case number } & \multicolumn{2}{|l|}{ OS } & \multicolumn{2}{|l|}{ DFS/RFS/bRFS } \\
\hline & & & $\begin{array}{l}\text { High } \\
\text { expression }\end{array}$ & $\begin{array}{l}\text { Low } \\
\text { expression }\end{array}$ & HR $(95 \% \mathrm{Cl})$ & p Value & HR (95\% Cl) & p Value \\
\hline Markou, 2013 & qRT-PCR & Mean & 47 & 57 & $0.41(0.18 \text { to } 0.94)^{\mathrm{M}}$ & 0.036 & $0.50(0.26 \text { to } 0.93)^{\mathrm{M}}$ & 0.030 \\
\hline Madden, 2013 & Microarray & NM & 291 & 290 & $0.77(0.61 \text { to } 0.97)^{\mathrm{U}}$ & 0.025 & NM & NM \\
\hline Kalogirou, 2013 & qRT-PCR & Median & 59 & 19 & NM & NM & $1.32(0.62 \text { to } 2.86)^{\mathrm{M}}$ & 0.472 \\
\hline Hulf, 2013 & qRT-PCR & Median & 106 & 43 & NM & NM & $0.50(0.28 \text { to } 0.90)^{\mathrm{M}}$ & 0.020 \\
\hline Hou, 2013 & qRT-PCR & Median & 41 & 61 & $0.24(0.11 \text { to } 0.56)^{\mathrm{M}}$ & 0.001 & NM & NM \\
\hline Hagman, 2013 & qRT-PCR & Median & NM & NM & $0.43(0.20 \text { to } 0.90)^{\mathrm{U}}$ & 0.030 & NM & NM \\
\hline Ratert, 2013 & qRT-PCR & Median & 25 & 15 & $0.28(0.07 \text { to } 1.11)^{U_{*}}$ & 0.070 & NM & NM \\
\hline Tran, 2013 & qRT-PCR & Mean & 49 & 49 & $2.08(1.25 \text { to } 3.57)^{U_{*}}$ & 0.005 & NM & NM \\
\hline Zhang, 2012 & qRT-PCR & Mean & NM & NM & $42.33(1.51 \text { to } 148.52)^{\mathrm{U}}$ & $<0.001$ & NM & NM \\
\hline Quesne, 2012 & LNA-ISH & NM & NM & NM & $0.79(0.64 \text { to } 0.97)^{\mathrm{U}}$ & 0.020 & NM & NM \\
\hline Le, 2012 & qRT-PCR & Mean & 21 & 21 & $1.23(0.45 \text { to } 3.37)^{\mathrm{U}}$ & 0.689 & $0.50(0.18 \text { to } 1.35)^{\mathrm{U}}$ & 0.169 \\
\hline Karaayvaz, 2012 & qRT-PCR & Mean & 24 & 15 & $2.65(1.11 \text { to } 6.25)^{\mathrm{M}}$ & 0.028 & NM & NM \\
\hline Hanna, 2012 & qISH & Mean & 113 & 93 & $0.51(0.26 \text { to } 0.997)^{\mathrm{M}}$ & 0.049 & NM & NM \\
\hline Radojicic, 2011 & qRT-PCR & Median & 38 & 49 & $1.45(0.65 \text { to } 3.23)^{\mathrm{U}_{*}}$ & 0.361 & $1.16(0.52 \text { to } 2.56)^{U_{*}}$ & 0.721 \\
\hline Schaefer, 2010 & qRT-PCR & Median & NM & NM & NM & NM & $0.37(0.10 \text { to } 1.40)^{\mathrm{M}}$ & 0.457 \\
\hline Childs, 2009 & RT-PCR & Mean & NM & NM & $0.40(0.18 \text { to } 0.88)^{\mathrm{M}}$ & 0.025 & $0.24(0.06 \text { to } 0.99)^{\mathrm{M}}$ & 0.049 \\
\hline Markou, 2008 & qRT-PCR & 2.00 & 31 & 17 & $1.27(0.52 \text { to } 3.13)^{U_{*}}$ & 0.610 & $1.32(0.62 \text { to } 2.86)^{U_{*}}$ & 0.476 \\
\hline
\end{tabular}

The source of $\mathrm{HR}$ and $95 \% \mathrm{Cl}$ is described as derived from univariate analysis (U) or multivariate analysis (M).

${ }^{*} \mathrm{HR}$ and $95 \% \mathrm{Cl}$ calculated from survival curves.

bRFS, biochemical recurrence-free survival; DFS, disease-free survival; HR, HR (high vs low); LNA-ISH, locked nucleic acid probe in situ hybridisation; NM, not mentioned; OS, overall survival; qISH, quantitative in situ hybridisation; qRT-PCR, quantitative real-time PCR; RFS, relapse-free survival. 
Table 3 Pooled HRs, 95\% Cls, and p values for OS or DFS/RFS stratified by ethnicity and main pathological type for overall and subgroup analyses

\begin{tabular}{|c|c|c|c|c|c|c|}
\hline \multirow[b]{2}{*}{ Subgroup } & \multicolumn{3}{|l|}{ os } & \multicolumn{3}{|c|}{ DFS/RFS } \\
\hline & $\overline{\mathbf{N}}$ & pHR (95\% Cl) & p Value & $\bar{N}$ & pHR (95\% Cl) & p Value \\
\hline Total & 14 & $0.82(0.59 \text { to } 1.15)^{\star}$ & 0.255 & 8 & $0.69(0.46 \text { to } 1.03)^{\star}$ & 0.067 \\
\hline \multicolumn{7}{|l|}{ Ethnic subtotal } \\
\hline Caucasian & 11 & $0.82(0.60 \text { to } 1.12)^{\star}$ & 0.214 & 7 & $0.71(0.46 \text { to } 1.11)^{\star}$ & 0.133 \\
\hline Asian & 3 & $1.76(0.20 \text { to } 15.74)^{\star}$ & 0.611 & 1 & 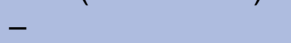 & - \\
\hline \multicolumn{7}{|l|}{ Disease subtotal } \\
\hline Breast cancer & 4 & $0.78(0.67$ to 0.91$) \dagger$ & 0.001 & 2 & $0.74(0.32 \text { to } 1.67)^{\star}$ & 0.463 \\
\hline Bladder cancer & 2 & $0.85(0.12 \text { to } 5.99)^{\star}$ & 0.869 & - & - & - \\
\hline NSCLC & 3 & $2.70(0.62 \text { to } 11.79)^{*}$ & 0.186 & 2 & $0.86(0.34 \text { to } 2.21)^{*}$ & 0.756 \\
\hline Prostate cancer & 1 & - & - & 3 & $0.67(0.32 \text { to } 1.41)^{*}$ & 0.287 \\
\hline \multicolumn{7}{|c|}{ Pathological type subtotal } \\
\hline AdenoCA & 9 & $0.85(0.60 \text { to } 1.19)^{\star}$ & 0.333 & 5 & $0.68(0.49$ to 0.94$) \dagger$ & 0.020 \\
\hline $\mathrm{SqCa}$ & 2 & $3.62(0.04 \text { to } 346.07)^{\star}$ & 0.581 & 1 & - & - \\
\hline \multicolumn{7}{|c|}{ Detected sample subtotal } \\
\hline Tissue & 13 & $0.80(0.56 \text { to } 1.14)^{*}$ & 0.221 & 7 & $0.71(0.46 \text { to } 1.11)^{*}$ & 0.133 \\
\hline Serum & 1 & - & - & 1 & - & - \\
\hline
\end{tabular}

\section{A Overall survival Ethnic subgroup}

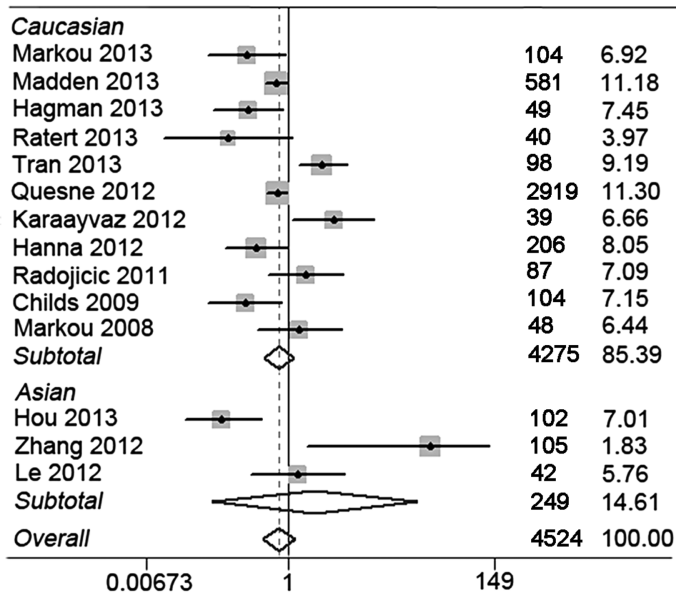

C

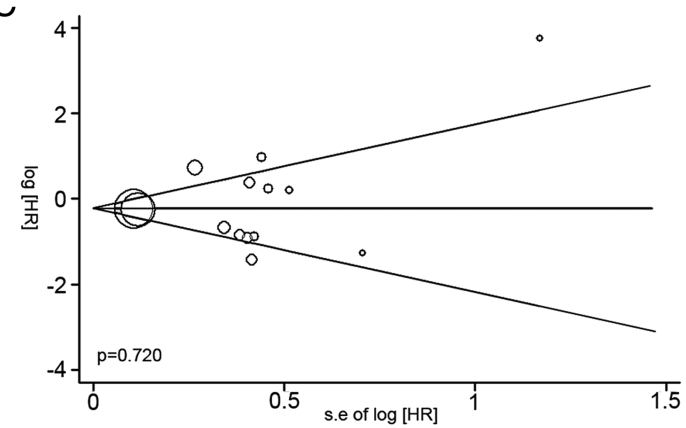

\section{B DFS/ RFS}

\section{Ethnic subgroup}

\section{N Weight}

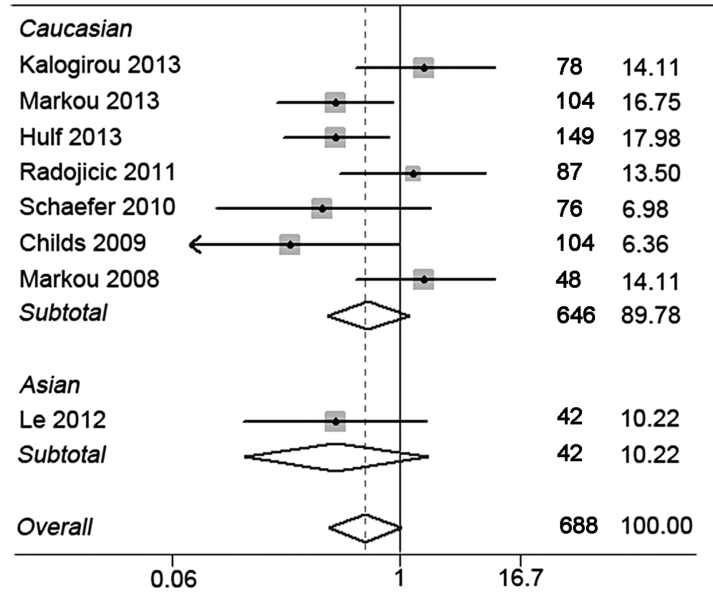

D

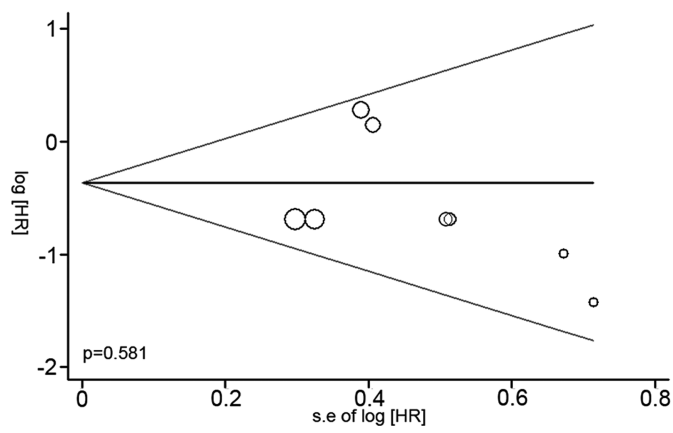

Figure 5 Forest plots of merged analyses of overall survival (OS) or DFS, disease-free survival (DFS)/relapse-free survival (RFS) in association with microRNA-205 expression and Begg's funnel plots of the publication bias. (A) Forest plots of overall and ethnic subgroup analyses of OS. Squares and horizontal lines represent study-specific HRs and $95 \% \mathrm{Cls}$, respectively. The areas of the squares correspond to weights, and the diamonds represent the overall HRs and $95 \% \mathrm{Cls}$. (B) Forest plots of overall and ethnic subgroup analyses of DFS/RFS. (C) Begg's funnel plots of the publication bias for overall merged analysis of OS. Each point represents a separate study. (D) Begg's funnel plots of the publication bias for the overall merged analysis of DFS/RFS. 
A Overall survival

Disease subgroup

Breast cancer

Markou 2013

Madden 2013

Radojicic 2011

Subtotal

Bladder cancer

Ratert 2013

Tran 2013

Subtotal

NSCLC

Zhang 2012
Le 2012

Markou 2008

Subtotal

Overall

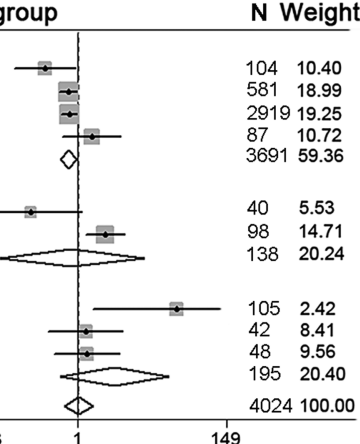

D DFS/RFS

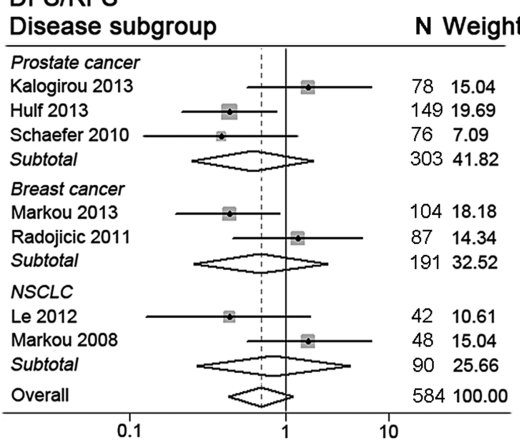

B Overall survival

Sample subgroup

Markou 2013

Hou 2013

Hagman 2013
Ratert 2013
Tran 2013

Zhang 2012

Kuesne 2012

Karaayvaz
Hanna 2012
Radojicic 2011

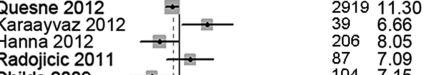

Childs 2009

Markou 2008
Subtotal

Serum

Le 2012

Subtotal

Overall

0.00673

1

E DFS/RFS

Sample subgroup

Tissue

Markou 2013

Hulf 2013

Radojicic 2011

Schaefer 2010

Childs 2009

Markou 2008

Subtotal

Serum

Le 2012

Subtotal

Overall

0.06

78

$\begin{array}{ll}78 & 14.11 \\ 104 & 16.75\end{array}$

14917.98

14917.98

$\begin{array}{ll}87 & 13.50 \\ 76 & 6.98\end{array}$

1046.36

16.7

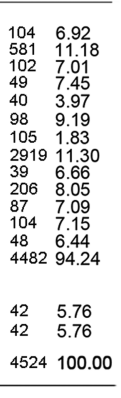

C Overall survival

Pathological subgroup

N Weight

Adenoca

Markou $2013 \longrightarrow-1048.49$

Madden $2013 \quad 581 \quad 14.09$

Hagman $2013 \quad 49 \quad 9.17$

$\begin{array}{lll}\text { Ratert } 2013 \quad 40 & 4.78 \\ \text { Tran } 2013 & 48 & 11.43\end{array}$

$\begin{array}{lll}\text { Tran } 2013 & 98 & 11.43 \\ \text { Quesne } 2012 & 2919 & 14.25\end{array}$

Karaayvaz 2012

Hanna $2012 \longrightarrow 2069.94$

Radojicic 2011

Subtotal 412389.03

SqCa

Zhang $2012 \quad \longrightarrow \quad \begin{array}{lll}0 & 205 & 2.18\end{array}$

Childs $2009 \quad 104 \quad 8.79$

Subtotal 20910.97

Overall 4332100.00

0.0028

F DFS/RFS

Pathological subgroup N Weight

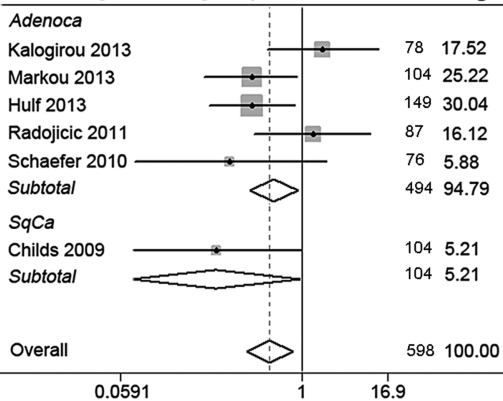

$\begin{array}{ll}48 & 14.11\end{array}$

64689.78

$42 \quad 10.22$

$42 \quad 10.22$

688100.00

Figure 6 Forest plots of merged analyses of OS or DFS/RFS in association with miRNA-205 expression for the different subgroups. (A) Forest plots of the merged analyses of OS in the different disease subgroups. Squares and horizontal lines correspond to the study-specific HRs and $95 \% \mathrm{Cls}$, respectively. The areas of the squares represent the weight, whereas the diamonds represent the summary HRs and $95 \%$ Cls. (B) Forest plots of the merged analyses of OS for the different disease subgroups. (C) Forest plots of the merged analyses of OS for the different sample subgroups. (D) Forest plots of the merged analyses of DFS/RFS for the different sample subgroups. (E) Forest plots of the merged analyses of DFS/RFS for the different pathological subgroups. (F) Forest plots of the merged analyses of DFS/RFS for the different pathological subgroups. Adenoca, adenocarcinoma; SqCa, squamous carcinoma; DFS, disease-free survival; RFS, relapse-free survival; NSCLC, non-small cell lung cancer; miRNA-205, microRNA-205; OS, overall survival.

respectively, as shown by symmetric funnel plots. Therefore, no evidence of publication bias was noted.

\section{DISCUSSION}

To identify prognostic biomarkers for patients with carcinoma, elaborate efforts have been invested in establishing reliable and convincing evidence. In the last decade, miRNAs have been considered to be potential biomarkers of cancer prognosis given their unique expression profiles in the tissues or serum of patients with cancer compared with normal individuals. MicroRNAs are more stably expressed than mRNAs and can be easily assessed by qRT-PCR. ${ }^{30}$ Recently, a number of miRNAs, acting as tumour-suppressors or oncogenes, have been shown to play important roles in tumourigenesis and cancer progression. ${ }^{31-33}$ Among them, miRNA-205 is considered to be important given its significant difference in expression between cancer and normal tissues.

This meta-analysis is the first systematic evaluation of the association between elevated miRNA-205 expression and the prognosis of patients with various cancers. The association of miRNA-205 expression with tumour prognosis may be partially attributed to its biological function.
Recent studies have reported that miRNA-205 is frequently deregulated in multiple tumour entities and involved in various crucial pathways, such as cell proliferation, apoptosis and angiogenesis, by mediating the expression of multiple oncogenes (figure 7). Hanna et $a l^{15}$ confirmed that miRNA-205 overexpression suppresses cell growth in vivo, induces cell senescence and reduces cell proliferation by downregulating adenovirus E2 promoter-binding transcription factor 1 (E2F1) in patients with melanoma. MicroRNA-205 also specifically activates the tumour suppressor genes IL24 and IL32 by targeting specific sites in their promoters ${ }^{7}$ and participates in the epithelial-mesenchymal transition by regulating zinc finger E-box-binding proteins (ZEB1/ZEB2) ${ }^{20}$

Our OS analysis revealed a pooled HR of 0.82 , demonstrating that increased miRNA-205 expression is associated with a favourable outcome; however, this result was not significant $(p=0.225)$. On the contrary, the pooled outcome in the DFS/RFS analysis indicated that increased miRNA-205 expression is predictive of a better prognosis, with an HR value of 0.69 , but this association was also statistically insignificant $(\mathrm{P}=0.067)$. Insignificant pooled outcomes may imply that the role of miRNA-205 in human tumours is complex and controversial. The 


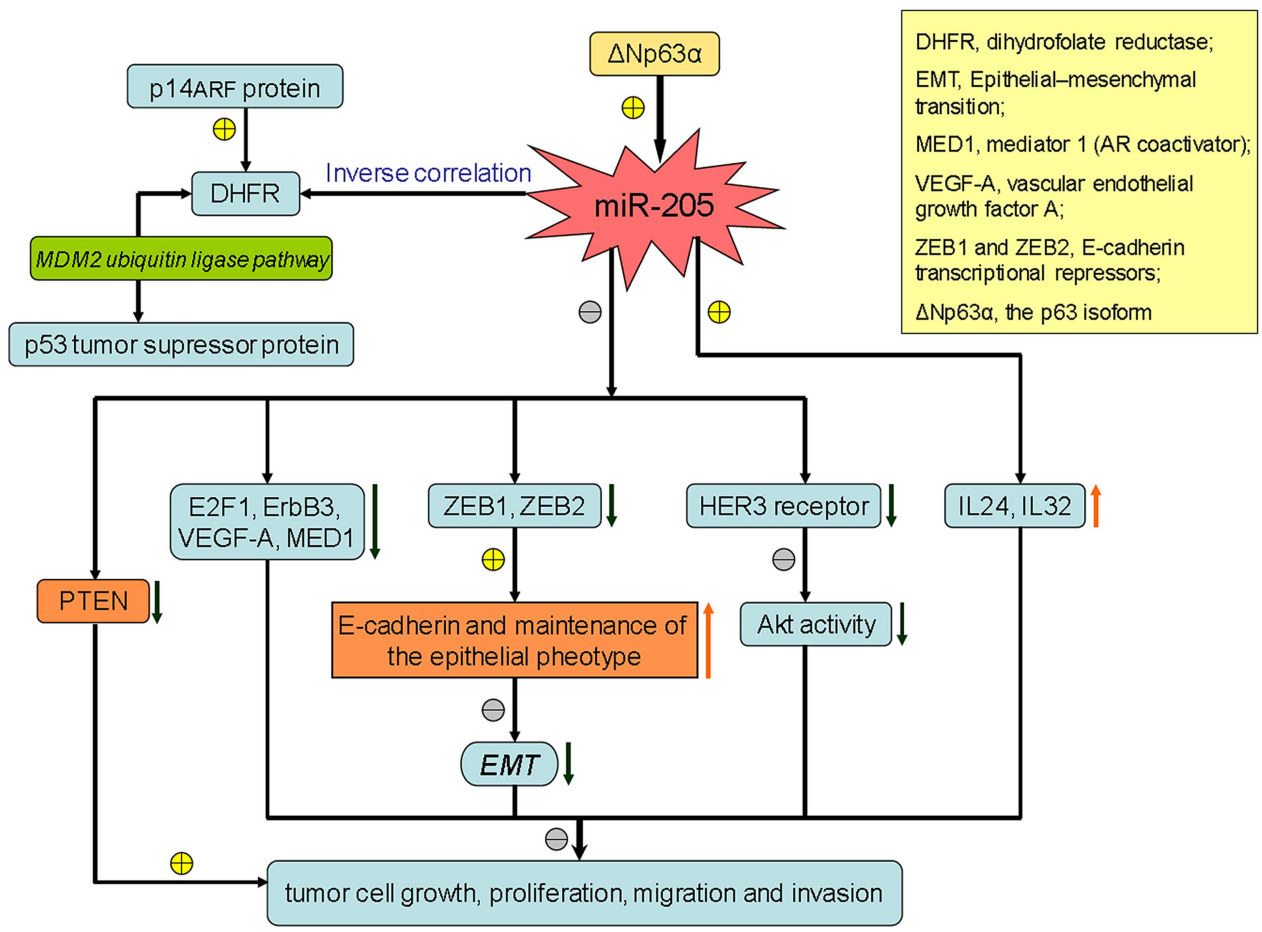

Figure 7 The complex roles of miRNA-205 in tumour growth, migration and invasion (miRNA-205, microRNA-205; PTEN, phosphatase and a tensin homolog on chromosome 10).

majority of studies have suggested that miRNA-205 is an important tumour suppressor. However, its oncogenic role has also been confirmed, which involves the inhibition of tumour suppressors, such as phosphatase and a tensin homolog on chromosome 10 (PTEN). Karaayvaz et $a l^{21}$ indicated that miRNA-205 and PTEN expression levels are inversely correlated in patients with endometrial cancer, implying a significant inhibitory mechanism of miRNA-205 in mediating PTEN expression.

Furthermore, in subgroup analyses based on the characteristics of the individual studies, we observed statistically significant outcomes in the OS of the breast cancer subgroup and the DFS/RFS of the adenoCA subgroup, with pooled HRs of 0.78 and 0.68 , respectively. Iroio et al described miRNA-205 as a new oncosuppressive gene in breast cancer, which interferes with the proliferative pathway mediated by the human epidermal growth factor receptor (HER) family. This group also provided experimental evidence suggesting that miRNA-205 improves a patient's response to specific anticancer therapies. ${ }^{33}$ These findings suggest that the detection of miRNA-205 expression in patients with breast cancer and adenoCA may be useful for prognosis prediction.

However, our conclusions should be considered with caution for the following five reasons. First, the power of the pooled HRs was not sufficiently strong even though our results indicate that miRNA-205 expression is significantly associated with the prognoses of breast cancer and adenoCA. Second, the statistical power of the association result of miRNA-205 with adenoCA was reduced as a result of five studies that were included in the DFS/RFS meta-analysis with a relatively small sample size of 494 patients. Moreover, three studies in the adenoCA subgroup exhibited statistically insignificant outcomes. Third, the lack of global miRNA-205 expression data makes it difficult to define a standard cut-off. Most of the current studies have established a median or mean value as the expression cut-off, and these values have varied. Therefore, the pooled outcome may be higher or lower than the actual value, which may have caused a bias in the results of the effectiveness of miRNA-205 as a prognostic factor. Fourth, heterogeneity existed in the total OS and DFS/RFS analyses. The heterogeneity of the population was likely due to the different characteristics of the patients, such as age, race, disease type and pathological type, as well as the cut-off value of miRNA-205 expression. For instance, when we stratified the OS studies according to disease type, the heterogeneity disappeared in the breast cancer subgroup ( $\mathrm{p}=0.199$, $\mathrm{I}^{2}=35.5 \%$ ). The heterogeneity was also reduced in the adenoCA subgroup when the DFS/RFS studies were classified by pathological type $\left(\mathrm{p}=0.116, \mathrm{I}^{2}=45.9 \%\right)$. Fifth, although miRNA-205 is considered an independent risk factor for carcinomas, ${ }^{5} 812$ recent studies have used a linear combination of several miRNAs rather than a unique miRNA to increase predictive power. Shiiyama et $a l^{84}$ demonstrated that the combined assessment of miRNA-9, miRNA-145, miRNA-150, miRNA-155 and miRNA-205 was more sensitive than the assessment of any of these miRNAs individually for distinguishing patients with metastatic melanoma from patients with nonmetastatic melanoma. Therefore, relevant studies are required to address the aforementioned problems before 
miRNA-205 may be used as a prognostic biomarker in clinical applications.

\section{CONCLUSIONS}

In summary, our findings suggest that miRNA-205 is a potential biomarker and that the detection of its expression in patients with breast cancer may allow for accurate prognostic predictions. Moreover, further investigation of the relationship between miRNA-205 expression and cancer prognosis is required to enable its future clinical application.

Acknowledgements The authors would like to thank the American Journal Experts for their professional editing and comments (Certificate Verification Key: 3676-A3BA-9A08-106A-EBE3). Moreover, the authors would like to thank the researchers of the primary studies.

Contributors JY and J-yZ conceived and designed the study. M-yS and N-hS collected the data. J-yZ, Z-ID and C-yX performed statistical analyses. J-yZ, $M-y S$ and $J Y$ drafted the manuscript. J-yZ and C-yX revised the manuscript. All authors have read and approved the final version.

Funding This work was funded by the Priority Academic Program Development of Jiangsu Higher Education Institutions (PAPD), grant no JX10231801.

\section{Competing interests None.}

Patient consent Obtained.

Ethics approval The ethical committee of Nanjing Medical University.

Provenance and peer review Not commissioned; externally peer reviewed.

Data sharing statement No additional data are available.

Open Access This is an Open Access article distributed in accordance with the Creative Commons Attribution Non Commercial (CC BY-NC 4.0) license, which permits others to distribute, remix, adapt, build upon this work noncommercially, and license their derivative works on different terms, provided the original work is properly cited and the use is non-commercial. See: http:// creativecommons.org/licenses/by-nc/4.0/

\section{REFERENCES}

1. Carthew RW, Sontheimer EJ. Origins and mechanisms of miRNAs and siRNAs. Cell 2009;136:642-55.

2. Lee RC, Feinbaum RL, Ambros V. The C. elegans heterochronic gene lin-4 encodes small RNAs with antisense complementarity to lin-14. Cell 1993;75:843-54.

3. Filipowicz W, Bhattacharyya SN, Sonenberg N. Mechanisms of posttranscriptional regulation by microRNAs: are the answers in sight? Nat Rev Genet 2008;9:102-14

4. Yang W, Lee DY, Ben-David Y. The roles of microRNAs in tumorigenesis and angiogenesis. Int J Physiol Pathophysiol Pharmacol 2011;3:140-55.

5. Hulf T, Sibbritt T, Wiklund ED, et al. Epigenetic-induced repression of microRNA-205 is associated with MED1 activation and a poorer prognosis in localized prostate cancer. Oncogene 2013;32:2891-9.

6. Markou A, Yousef GM, Stathopoulos E, et al. Prognostic significance of metastasis-related microRNAs in early breast cancer patients with a long follow-up. Clin Chem 2014;60:197-205.

7. Majid S, Dar AA, Saini S, et al. MicroRNA-205-directed transcriptional activation of tumor suppressor genes in prostate cancer. Cancer 2010;116:5637-49.

8. Hou SX, Ding BJ, Li HZ, et al. Identification of microRNA-205 as a potential prognostic indicator for human glioma. J Clin Neurosci 2013;20:933-7.

9. Orang AV, Safaralizadeh R, Hosseinpour Feizi MA, et al. Diagnostic and prognostic value of miR-205 in colorectal cancer. Asian Pac J Cancer Prev 2014:15:4033-7.
10. Zhang G, Hou X, Li Y, et al. MiR-205 inhibits cell apoptosis by targeting phosphatase and tensin homolog deleted on chromosome ten in endometrial cancer Ishikawa cells. BMC Cancer 2014;14:440.

11. Madden SF, Clarke C, Gaule P, et al. BreastMark: an integrated approach to mining publicly available transcriptomic datasets relating to breast cancer outcome. Breast Cancer Res 2013;15:R52.

12. Hagman Z, Haflidadóttir BS, Ceder JA, et al. miR-205 negatively regulates the androgen receptor and is associated with adverse outcome of prostate cancer patients. $\mathrm{Br} J$ Cancer 2013;108:1668-76.

13. Ratert $\mathrm{N}$, Meyer HA, Jung M, et al. miRNA profiling identifies candidate mirnas for bladder cancer diagnosis and clinical outcome. J Mol Diagn 2013;15:695-705.

14. Quesne JL, Jones J, Warren J, et al. Biological and prognostic associations of miR-205 and let-7b in breast cancer revealed by in situ hybridization analysis of micro-RNA expression in arrays of archival tumor tissue. J Pathol 2012;227:306-14.

15. Hanna JA, Hahn L, Agarwal S, et al. In situ measurement of miR-205 in malignant melanoma tissue supports its role as a tumor suppressor microRNA. Lab Invest 2012;92:1390-7.

16. Schaefer A, Jung M, Mollenkopf HJ, et al. Diagnostic and prognostic implications of microRNA profiling in prostate carcinoma. Int $J$ Cancer 2010;126:1166-76.

17. Childs G, Fazzari M, Kung G, et al. Low-level expression of microRNAs let-7d and miR-205 are prognostic markers of head and neck squamous cell carcinoma. Am J Pathol 2009;174:736-45.

18. Kalogirou C, Spahn M, Krebs M, et al. MiR-205 is progressively down-regulated in lymph node metastasis but fails as a prognostic biomarker in high-risk prostate cancer. Int $\mathrm{J} \mathrm{Mol} \mathrm{Sci}$ 2013;14:21414-34

19. Zhang YK, Zhu WY, He JY, et al. miRNAs expression profiling to distinguish lung squamous-cell carcinoma from adenocarcinoma subtypes. J Cancer Res Clin Oncol 2012;138:1641-50.

20. Tran MN, Choi W, Wszolek MF, et al. The p63 protein isoform $\Delta \mathrm{Np} 63 \alpha$ inhibits epithelial-mesenchymal transition in human bladder cancer cells: role of MIR-205. J Biol Chem 2013;288:3275-88.

21. Karaayvaz M, Zhang $\mathrm{C}$, Liang $\mathrm{S}$, et al. Prognostic significance of miR-205 in endometrial cancer. PLOS ONE 2012;7:e35158.

22. Radojicic J, Zaravinos A, Vrekoussis T, et al. MicroRNA expression analysis in triple-negative (ER, PR and Her2/neu) breast cancer. Cell Cycle 2011;10:507-17.

23. Markou A, Tsaroucha EG, Kaklamanis L, et al. Prognostic value of mature microRNA-21 and microRNA-205 overexpression in non-small cell lung cancer by quantitative real-time RT-PCR. Clin Chem 2008;54:1696-704.

24. Le HB, Zhu WY, Chen DD, et al. Evaluation of dynamic change of serum miR-21 and miR-24 in pre- and post-operative lung carcinoma patients. Med Oncol 2012;29:3190-7.

25. Moher D, Liberati A, Tetzlaff J, et al. Preferred reporting items for systematic reviews and meta-analyses: the PRISMA statement. BMJ 2009;339:b2535.

26. Williamson PR, Smith CT, Hutton JL, et al. Aggregate data meta-analysis with time-to-event outcomes. Stat Med 2002;21:3337-51.

27. Tierney JF, Stewart LA, Ghersi D, et al. Practical methods for incorporating summary time-to-event data into meta-analysis. Trials 2007;8:16.

28. DerSimonian R, Laird N. Meta-analysis in clinical trials. Control Clin Trials 1986;7:177-88.

29. Egger M, Davey Smith G, Schneider M, et al. Bias in meta-analysis detected by a simple, graphical test. BMJ 1997;315:629-34.

30. Ferracin M, Veronese A, Negrini M. Micromarkers: miRNAs in cancer diagnosis and prognosis. Expert Rev Mol Diagn 2010;10:297-308.

31. le Sage C, Nagel R, Egan DA, et al. Regulation of the p27(Kip1) tumor suppressor by miR-221 and miR-222 promotes cancer cell proliferation. EMBO J 2007;26:3699-708.

32. Law PT, Ching AK, Chan AW, et al. miR-145 modulates multiple components of the insulin-like growth factor pathway in hepatocellular carcinoma. Carcinogenesis 2012;33:1134-41.

33. Iorio MV, Casalini $P$, Piovan $C$, et al. microRNA-205 regulates HER3 in human breast cancer. Cancer Res 2009;69:2195-200.

34. Shiiyama R, Fukushima S, Jinnin M, et al. Sensitive detection of melanoma metastasis using circulating microRNA expression profiles. Melanoma Res 2013;23:366-72. 